\section{Central nervous system diseases associated with blood brain barrier breakdown - A Comprehensive update of existing literature}

\author{
Rajib Dutta* \\ MD, Neurology, India
}

\section{Abstract}

Blood vessels that supply and feed the central nervous system (CNS) possess unique and exclusive properties, named as blood-brain barrier (BBB). It is responsible for tight regulation of the movement of ions, molecules, and cells between the blood and the brain thereby maintaining controlled chemical composition of the neuronal milieu required for appropriate functioning. It also protects the neural tissue from toxic plasma components, blood cells and pathogens from entering the brain. In this review the importance of BBB and its disruption causing brain pathology and progression to different neurological diseases like Alzheimer's disease (AD), Parkinson's disease (PD), Amyotrophic lateral sclerosis (ALS), Huntington's disease (HD) etc. will be discussed.

\section{More Information}

\author{
*Address for Correspondence: Rajib Dutta, \\ MD Neurology, India, \\ Email: rajibdutta808@gmail.com \\ Submitted: 22 April 2020 \\ Approved: 24 August 2020 \\ Published: 25 August 2020 \\ How to cite this article: Dutta R. Central \\ nervous system diseases associated with blood \\ brain barrier breakdown -A Comprehensive \\ update of existing literature. J Neurosci Neurol \\ Disord. 2020; 4: 053-062.
}

DOI: 10.29328/journal.jnnd.1001035

ORCiD: orcid.org/0000-0002-6129-1038

Copyright: (c) 2020 Dutta R. This is an open access article distributed under the Creative Commons Attribution License, which permits unrestricted use, distribution, and reproduction in any medium, provided the original work is properly cited.

Keywords: Blood brain barrier; Central nervous system; Neuronal functioning; Brain pathology; Neurological disease

W) Check for updates

(9) OPEN ACCESS

\section{Abbreviations}

CNS: Central Nervous System; BBB: Blood Brain Barrier; NVU: Neurovascular Unit; Smcs: Smooth Muscle Cells; VEGF: Vascular Endothelial Growth Factor; CBF: Cerebral Blood Flow; AD: Alzheimer's Disease; CSF: Cerebrospinal Fluid; S1P: Sphingosine-1-Phosphate; A : Amyloid Beta; MMP: Matrix Metalloproteinases; Timps: Tissue Inhibitors of Metalloproteinases; PD: Parkinson's Disease; A-Syn: Alpha-synuclein; Evs: Extracellular Vesicles; 6-OHDA: 6-Hydroxydopamine; RBC: Red Blood Cells; MPTP: 1-Methyl4-Phenyl-1,2,3,6-Tetrahydropyridine; Rcbf: Regional Cerebral Blood Flow ; DA: Dopamine; HD: Huntington's Disease; MRI: Magnetic Resonance Imaging; TJ: Tight Junction; GLUT1: Glucose Transporter 1; SOD: Superoxide Dismutase; ALS: Amyotrophic Lateral Sclerosis; PCAM: Platelet Cell Adhesion Molecule; GFAP: Glial Fibrillary Acidic Protein; IL: Interleukin; CCL: Chemokine (C-C Motif) Ligand; CCR: C-C Chemokine Receptor; CXCL: C-X-C Motif Chemokine Ligand; CXCR: C-X-C Chemokine Receptor; OCB: Oligoclonal Band; TARDBP: TAR DNA-Binding Protein; ANG: Angiogenin; mRNA: Messenger RNA; CEC: Circulating Endothelial Cells; MS: Multiple Sclerosis; OPC: Oligodendrocyte Precursor Cell; TSC: Total Sodium Concentration; ADC: Apparent Diffusion Coefficient; HIV: Human Immunodeficiency Virus; HAD: HIV-1-Associated Dementia; HAND: HIV-Associated Neurocognitive Disorder;
PDGF: Platelet-Derived Growth Factor; CTE: Chronic Traumatic Encephalopathy; TBI: Traumatic Brain Injury; mTBI: Mild Traumatic Brain Injury; bTBI: Blast-Induced Traumatic Brain Injury; P-T: P-Tau; Igg: Immunoglobulin G; HLA: Human Leukocyte Antigen; MIF: Macrophage Migration Inhibitory Factor; MCP: Monocyte Chemoattractant Protein; ICAM: Intercellular Adhesion Molecule; VCAM: Vascular Cell Adhesion Molecule; ICH: Intracerebral Hemorrhage; MAPK: Mitogen-Activated Protein Kinases; SAH: Subarachnoid Hemorrhage; NADPH: Nicotinamide Adenine Dinucleotide Phosphate Hydrogenase; TLE: Temporal Lobe Epilepsy; CD: Cluster Of Differentiation; SE: Status Epilepticus; TGF-B: Transforming Growth Factor Beta; CDK: Cyclin-Dependent Kinase; HBMEC: Human Brain Microvascular Endothelial Cells

\section{Introduction}

Blood vessels of CNS are continuous, non-fenestrated, and also contain a series of additional properties that allow them to tightly regulate the movement of molecules, ions, and cells between the blood and the CNS $[1,2]$. This heavy restriction allows endothelial cells of BBB to tightly regulate CNS homeostasis, required for proper neuronal function, as well as protect the CNS from toxins, pathogens, inflammation, injury, and disease [3]. The restrictive nature of the BBB is also known to provide an obstacle for drug delivery to the 
CNS. Major efforts have been made to generate methods to modulate or bypass the BBB for delivery of therapeutics [4].

Under physiological conditions, the human brain receives $20 \%$ of the cardiac output and uses $20 \%$ of the body's oxygen and glucose [5]. Cerebral blood vessels follows the major brain circuits tasked with sensation, memory, and motion suggesting that the cerebrovascular system plays an important role in normal CNS functioning [6-8]. Neurovascular coupling and energy substrate used by brain is through the BBB [6]. BBB permeability, neurovascular coupling, cell-matrix interactions, neurotransmitter turnover, angiogenesis and neurogenesis are controlled by neurovascular unit (NVU) $[5,6]$.

NVU comprises of vascular cells [e.g, endothelium and mural cells including pericytes and smooth muscle cells (SMCs)], glia (e.g, astrocytes, microglia), and neurons $[9,10]$. The BBB is centrally positioned within the NVU and is formed by a monolayer of tightly-sealed endothelial cells along the vascular tree expressing low paracellular and transcellular permeability $[8,11,12]$. BBB formation during embryogenesis is complex and is dependent on various signaling molecules like VEGF [13], wnt [14],sonic hedgehog [15], platelet derived growth factor-BB-platelet derived growth factor receptor beta- signaling [16], axon guidance molecules [17], and BBB specific components [8]. BBB maturation and its maintenance are controlled by neuronal activity-induced vascular plasticity, microRNAs and exosomal regulation, apicobasal polarity of BBB endothelium, vascular cell lineages, immunoregulation of the BBB [18], and breakdown [19].

In disease states, BBB breakdown and dysfunction leads to leakage of harmful blood components into the CNS, cellular infiltration, and aberrant transport and clearance of molecules [8,12], which is associated with cerebral blood flow (CBF) reductions and dysregulation [7], thereby contributing to neurological deficits.

\section{Diseases associated with $\mathrm{BBB}$ dysfunction}

\section{Chronic neurodegenerative diseases}

Alzheimer's disease: Evidences suggest role of microbiome in disruption of $A \beta$ metabolism/clearance, increased permeability of the $\mathrm{BBB}$, modulation of the neuroinflammatory response, inhibition of hippocampal neurogenesis highlighting the brain gut microbiome axis and potential role of gut microbiota in AD [20]. Many studies supported the idea that BBB dysfunction as a cause and consequence of $\mathrm{AD}$ [21-23]. Ujiie, et al. reported that BBB permeability precedes senile plaque formation in an $\mathrm{AD}$ mice model [24]. Wu, et al. recently showed the role of BBB in lead induced AD-like pathology [25]. A recent study by Shin and colleagues also reported BBB dysfunction in a 3D human neural cell culture microfluidic in vitro model of AD [26]. Another study assessed the relationship between levels of cerebrospinal fluid (CSF) soluble platelet-derived growth factor receptor $\beta$ (sPDGFR $\beta$ ), CSF albumin and CSF/ serum albumin ratio, reduced CSF $A \beta 42$ and elevated CSF total and phosphorylated tau in AD. They found a positive association between pericyte injury and BBB breakdown which is related to the severity of AD pathology [27]. One study looked at the expression of enzymes implicated in ceramide and sphingolipid metabolism and deregulation of de novo ceramide biosynthesis and S1P metabolism in liver and brain of mice with hyperhomocysteinemia. These metabolites can pass through BBB, exert neurotoxic responses, increase pro-inflammatory cytokines and can cause AD like neurodegeneration [28]. He, et al. looked at the link of vascular risk factors, metabolic syndrome, BBB disruption and risk of AD. They found out altered expression of lowdensity lipoprotein receptor-related protein 1 and receptor for advanced glycation end products at the microvascular endothelial cells dysregulate $A \beta$ transport across the BBB. Altered brain insulin signaling, insulin resistance, dyslipidemia and white matter lesions contribute to tau and $\mathrm{A} \beta$ pathogenesis [29]. Matrix metalloproteinases (MMP) control the functions of a number of signaling and scaffolding molecules involved in BBB disruption and neuronal death. MMPs and their physiological inhibitors, tissue inhibitors of metalloproteinases (TIMPs), interact at the molecular and cellular level thereby initiating neurodegenerative processes like AD [30].

Parkinson's disease: It may be accepted that BBB and leakage of serum component into the brain could lead to neurodegeneration in Parkinson's disease (PD) [31]. Pathophysiology of PD circles around formation, transmission and aggregation of toxic $\alpha$-synuclein ( $\alpha$-syn). Recent evidence has suggested about the role of extracellular vesicles (EVs) in the transport of $\alpha$-syn between brain regions. One study demonstrated red blood cells (RBC) produce $\alpha$-syn-rich EVs, which can cross BBB under inflammatory conditions initiated by administration of lipopolysaccharide peripherally. Authors believe these EVs arising in periphery might initiate or contribute to CNS $\alpha$-syn-related pathology [32]. Another study found out an altered BBB has a significant contribution to brain iron accumulation and neuroinflammation in the 6-hydroxydopamine (6-OHDA) rat model of PD [33].

A recent clinical trial used histologic markers of serum iron, protein, RBC extravasation to show a significant increased permeability of $\mathrm{BBB}$ in the postcommissural putamen of PD patients [34]. Pericytes are known to be a key cellular regulator of the BBB. It was shown in a rat model that monomeric $\alpha$-synuclein-activated pericytes may contribute to BBB breakdown in patients with PD [35]. One study looked at the role of matrix metalloproteinase-3 (MMP-3) in the loss of dopaminergic (DA) neurons in the nigrostriatal pathway in a 1-methyl-4-phenyl-1,2,3,6-tetrahydropyridine (MPTP) mouse model of PD. They found MMP-3 can play an important role in PD patients with neuroinflammatory changes and BBB breakdown [36]. 
One study looked at the permeability of BBB in basal ganglia and found out levodopa induced severity of dyskinesia correlate with regional flow-metabolism dissociation and increased BBB permeability within areas of active microvascular remodeling [37]. Ohlin, et al. looked at the impact of levodopa treatment on regional cerebral blood flow ( $\mathrm{rCBF}$ ) and metabolism in the basal ganglia in a rat model of PD. They found out increases in $\mathrm{rCBF}$ on levodopa may be accompanied by BBB hyperpermeability in the most affected regions [38].

Huntington's disease: A study by Vilaregutk, et al. looked at the role of MMPs -2 and -9 in BBB breakdown in the striatal lesions induced by the systemic administration of 3-nitropropionic acid rat model of Huntington's disease (HD). They found out there is a bigger role of BBB disruption by MMP-9 in the striatal injured areas [39]. A recent study used 3- and 7-Tesla magnetic resonance imaging (MRI) as well as postmortem tissue analyses to assess blood vessel impairments in HD patients. The following findings were further investigated in the R6/2 mouse model using in situ cerebral perfusion, histological analysis, western blotting, as well as transmission and scanning electron microscopy. The authors reported increase in blood density, reduction in blood vessel diameter and BBB leakage in both the mice model and HD patients [40]. Some other studies also reported about the evidence BBB disruption in HD [41-44].

Amyotrophic lateral sclerosis: Animal model studies have reported previously about the occurrence blood spinal cord barrier breakdown before motor neuron injury [45-49]. BBB breakdown with decreased levels of IgG, tight junction (TJ) proteins, GLUT1 reduction and perivascular hemosiderin deposits has been shown to precede motor neuron loss, neuroinflammation and motor impairments in SOD1 mutants $[46,48,49]$. A study showed early BBB disruption in SOD1G93A rats at a pre-symptomatic stage [50]. Contrary to a neuroimaging study which did not find any change in the integrity of BBB [51], several studies reported disrupted BBB in SOD1G93A mice [52-56].

Microvascular BBB abnormalities was shown in a postmortem study of a sporadic ALS patient [57]. A study which looked at the damage in the neurovascular unit in BBB, reported a possible activation of MMP-9 in ALS patients and ALS model mice. This damage occurred prior to motor neuron degeneration. They also reported on the dissociation between the PCAM-1-positive endothelium and GFAP-positive astrocyte foot processes in both humans and the animal model of ALS [46].

A recent case-control study reported the association increased CSF homocysteine with BBB disruption in ALS patients [58]. A study reported interleukin- $1 \beta$ Induces BBB disruption by downregulating sonic hedgehog in astrocytes. They also found IL- $1 \beta$ increased astrocytic production of pro- inflammatory chemokines such as CCL2, CCL20, and CXCL2, which induce immune cell migration and exacerbate $\mathrm{BBB}$ disruption and neuroinflammation [59]. One study reported ALS patients with OCBs in CSF may harbor mutations in disease-causing genes. The authors also speculated that mutations in both TARDBP and ANG genes may disrupt BBB and cause neuroinflammation [60].

One study reported lost endothelium integrity by decreased mRNA transcription of tight junction proteins in autopsied human spinal cords from both sporadic and familial forms of ALS [61]. Circulating endothelial cells (CECs) in the peripheral blood are associated with endothelium damage. One study quantified the CEC levels in whole blood smears from ALS patients with moderate stage ((M)ALS), severe stage ((S)ALS), and healthy controls by CD146 expression using immunocytochemistry. They found a significant reduction of CECs in (M)ALS and (S)ALS patients. The authors think endothelial damage and/or impaired endothelium repair may occur in ALS leading to BBB disruption [62].

Multiple sclerosis: MS is an autoimmune and neurodegenerative disease in which the myelin sheath surrounding axons is attacked by immune cells, including leukocytes, $\mathrm{T}$ cells, $\mathrm{B}$ cells, and peripheral macrophages that enter the brain through a disrupted BBB [63]. Neuroimaging studies using gadolinium enhancement have established that BBB disruption is an early feature of MS pathogenesis [64]. IL17A is associated with the breakdown of the BBB in relapsingremitting MS [65].

Perivascular OPCs can disrupt the BBB, interfere with astrocyte endfeet and endothelial tight junction integrity, resulting in altered vascular permeability and an associated CNS inflammation. Aberrant OPC perivascular migration responsible for BBB disruption in MS through defective oligodendroglial vascular interaction [66].

Human brain endothelial CXCR2 may contribute to BBB disturbance under inflammatory conditions with increased CXCL5 and CXCL8 expression, where CXCR2 may also represent a novel pharmacological target for blood-brain barrier stabilization. The study looked at the immunohistochemistry of brain biopsies from two patients with active MS which revealed upregulation of endothelial CXCR2 compared to healthy control tissue [67].

At the BBB, claudin-5 is the most enriched $\mathrm{Tj}$ protein and its dysfunction has been implicated in MS [68]. Another study reported downregulation of claudin-11 expression is associated with BBB disintegration in MS [69]. A recent study looked at the temporal evolution of acute MS lesions on serial sodium MRI during 4 weeks after the initial presentation. Quantitative assessment of total sodium concentration (TSC) and ADC was performed. They reported lesions with a reduction of the ADC sodium levels were almost normal and precede signs of BBB breakdown [70]. 
MRI changes suggestive of BBB disruption were also found in normally appearing white matter before enhancing lesions [71] and in non-enhancing areas in MS [72]. Disruption of BBB extend to the initial stages of the disease as evidenced at the onset of optic neuritis who develops MS in future [73]. A recent study on relapsing remitting MS looked at the circulating factors in blood that induce physiological and biochemical alterations to the BBB. Disruption of BBB endothelium in MS may be due to metabolic dysregulation, leading to increased permeability [74]. One study reported expression of fibronectin by activated astrocytes at acute MS lesions, reflecting the tissue remodeling at sites of $\mathrm{BBB}$ breakdown [75].

\section{Other chronic neurodegenerative diseases}

HIV-Associated Neurocognitive Disorder: HIV-1positive individuals today still develop HIV-1-associated dementia (HAD) and neurocognitive impairment in spite of good prognosis with antiretroviral therapy [76,77]. Imbalances between MMPs and TIMPs are involved in BBB disruption and are implicated in the pathogenesis of HAND in HIV-1 patients [78]. HIV-1-infected cells both in CNS and periphery can give rise to increased levels of viral proteins, including Nef, Tat, gp120 and host inflammatory mediators like cytokines and chemokines which can affect the integrity and permeability of BBB [79]. An animal study involving HIV Tg26 mice was associated with increased expression of PDGF$\mathrm{BB}$ in isolated micro vessels in brain with loss of pericytes and subsequent breach of BBB [80].

Chronic traumatic encephalopathy: Chronic traumatic encephalopathy (CTE) is associated with repeated mild traumatic brain injury (TBI). It is reported from all profession right from soccer, boxing, war veterans, and wrestling. A case of CTE was associated with disruption of BBB in regions of dense perivascular p-tau accumulation [81]. A recent study also provided evidence of BBB disruption in regions of intense perivascular $\mathrm{p}-\tau$ deposition in a former professional boxer diagnosed with CTE and schizophrenia. This p- $\tau$ deposition was linked to loss of tight junction protein claudin-5 and enhanced extravasation of endogenous blood components such as fibrinogen and IgG [82]. Upregulation of cleavedcaspase-3 is associated with accumulation of caspase-3cleaved tau following chronic TBI, suggesting role of apoptosis and neuroinflammation in delayed BBB damage [83]. Repetitive TBI can lead to end stage CTE. Several mechanisms responsible are elevated concentration of both tau proteins, human leukocyte antigen (HLA) class I proteins, macrophage entry into brain parenchyma from disruption of BBB and microglial activation at the base of the sulci $[84,85]$.

\section{Acute neurological disorders}

Stroke: Dysfunction of BBB is a prominent pathological feature of both ischemic and hemorrhagic stroke and is typically associated with poor outcome [86-88]. Ischemic stroke exhibits extravasation of blood-borne cells, chemicals, and fluid into brain parenchyma across the impaired BBB as a result of increased paracellular and transcellular permeability and endothelial degeneration [89]. Astrocytes, an important component of the BBB, promotes BBB breakdown in subjects with acute ischemic stroke by secreting inflammatory factors [90].Several factors which determine BBB permeability followed by breakdown are mitochondrial bioenergetics, microRNAs, MMPs (MMP-2 and MMP-9), cytokines (IL-1 $\beta$, MIF, IL-9), chemokines (MCP-1), immune cells, adhesion proteins (ICAM-1, VCAM-1) [91,94]. Recent studies support the role of CCL2 in BBB disruption following ICH through CCL2-CCR2-p38 MAPK pathway [92]. Dysfunction of tight junctions and endothelial cells is associated with BBB disruption following aneurysmal SAH. Several molecular targets have been linked, including receptor tyrosine kinase ErbB4, lipocalin-2, netrin-1, toll-like receptor 4, tropomyosinrelated kinase receptor B [93].

Traumatic brain injury: BBB disruption is a known consequence of TBI and is associated with poorer outcomes [101]. A study on animal model reported BBB alterations after TBI occur in two phases, first occurring within 4-6 hours of tissue damage and the second 3 days after injury in the injury site cortex and the ipsilateral hippocampus. Brain edema peaks at 24 hours after controlled cortical impact brain injury and declines after day 3 [95]. Another study which looked at the BBB permeability in a mouse model of TBI reported soon after trauma, both large and small molecules are able to enter the brain in and around the site of injury. BBB restriction to large (protein-sized) molecules is restored by $4-5 \mathrm{~h}$ after injury. However, smaller molecules (286-10,000 Da) are still able to enter the brain as long as 4 days postinjury [96]. However, Hay, et al. reported post TBI, BBB disruption can persist over years. Serum proteins like IgG and fibrinogen, both markers of BBB disruption were elevated that died in acute phase as well as those survived for a year [102]. Another study reported increased fibrinogen in human brain 6-72 $\mathrm{h}$ following severe TBI [103].

A recent study which looked at the BBB integrity causing TBI after exposure to blast overpressure reported impairment in BBB [97]. A recent study reported interaction of several inflammatory mechanisms causing persistent BBB disruption in mTBI with hypertension [98]. Oxidative stress is a major causative factor in the BBB breakdown in the sub-acute stages of blast-induced traumatic brain injury (bTBI). NADPH oxidase mediated oxidative stress leads to enhanced BBB permeability in bTBI through MMP activation [99]. Post TBI astrocyte-derived vascular permeability factors including vascular endothelial growth factors, MMP, nitric oxide, glutamate and endothelin-1, enhance BBB permeability leading to BBB disruption [100]. Several biomarkers has been linked to BBB disruption like CSF/serum albumin ratio [104], tight junction proteins $[105,106]$, S100B $[107,108]$, Plasmasoluble prion protein $(\mathrm{PrPc})[109,110]$. 
Epilepsy: The interrelationship between disruption of BBB and epilepsy are complex. Dysfunction of BBB may both lead to seizures and can be induced by epileptic activity [112]. BBB disruption positively correlates with seizure frequency and is independent of neuronal loss [109]. Persistent leakage of serum IgG in the interstitial space and their uptake by neurons may lead to hypoperfusion and in neuronal dysfunction occurring in temporal lobe epilepsy (TLE) [110].

Recent study looked at activation of innate immune system in TLE. They reported persistent increase in monocyte infiltration, microglia, and perivascular macrophage activation in both epileptogenic human and rat hippocampus in relation to seizure activity and BBB dysfunction. The expression of CD68 and CCL2 was related to the duration of epilepsy and type of pathology in humans, whereas the expression of CD68, CCL2, and the perivascular macrophage marker CD163 was related to the duration of the initial insult and to the number of spontaneous seizures in rats. One interesting finding was the number of CD163-positive perivascular macrophages was also positively correlated to BBB dysfunction in chronic epileptic rats [120].

BBB dysfunction is a prominent finding in status epilepticus (SE). This is observed within the $1^{\text {st }}$ hour of SE and may last for months in epileptogenic brain regions. BBB disruption have a role in astroglial dysfunction, neuroinflammation, increasing neural excitability, reduction of seizure threshold, excitatory synaptogenesis, impaired plasticity, and thereby generating epileptogenic activity. Transforming growth factor beta (TGF- $\beta$ ) proinflammatory pathway is involved in BBB dysfunction inducing neurovascular dysfunction activated by extravasation of serum albumin into the brain [111].

TBI and underlying inflammatory cascade involved in BBB breakdown can trigger epilepsy $[113,114,118]$. Permanent changes in permeability of BBB is associated with progression and treatment resistance $[115,116]$. One study reported about endothelial cyclin-dependent kinase 5 (CDK5), a key central regulator of neuronal excitability. Endothelial-specific Cdk5 knockout led to spontaneous seizures in a mice model. Increased endothelial chemokine (C-X-C motif) ligand 1 (Cxcl1) expression, decreased astrocytic glutamate reuptake through the glutamate transporter 1 (GLT1), and increased glutamate synaptic function was seen in the knockout mice [117].

In genetic epilepsies, such as tuberous sclerosis complex (TSC) and other related epileptogenic developmental pathologies, there is an association between the underlying gene mutation, BBB dysfunction, and perivascular inflammation, but a clear direct evidence is lacking [119].

\section{Miscellanous disorders}

Schizophrenia: A weak association exists between schizophrenia, Tj protein and claudin-5. Claudin-5 is expressed in endothelial cells forming part of the BBB. Schizophrenia occurs in $30 \%$ of individuals with $22 \mathrm{q} 11$ deletion syndrome (22q11DS), a population who are haploinsufficient for the claudin-5 gene. It was reported that targeted adeno-associated virus-mediated suppression of claudin- 5 in the mouse brain results in localized BBB disruption and behavioural changes. It was also showed that anti-psychotic medications dosedependently increase claudin-5 expression in vitro and in vivo, whereas post mortem reports in the brain of schizophrenic patients had discontinuous expression of claudin- 5 compared to age-matched controls [121].

A recent study reported general OSOS (overall severity of schizophrenia) dimension and a single-group negative symptom dimension are associated with a breakdown of BBB [122]. Another study looked at the association of deficit schizophrenia and leaky paracellular, transcellular, and vascular barriers in the BBB. They concluded deficit syndrome is a result of BBB dysfunction secondary to breakdown of paracellular and vascular pathways [123].

Meningitis: When the meninges are inflamed, the BBB may be disrupted. This disruption may increase the penetration of various substances into the brain depending on the permeability [124].

In a study, it was reported that significant induction of PDGF-B and ICAM-1 exists in meningitic E. coli mouse as well as monolayer hBMECs models. The increase of PDGF-B may directly enhance the BBB permeability by decreasing the expression of $\mathrm{Tj}$ proteins, and the upregulation of ICAM1 contributing to neutrophils or monocytes recruitment as well as neuroinflammation in response to meningitic E. coli infection [125].

Streptococcus equi subsp. zooepidemicus (SEZ) is a zoonotic pathogen which causes meningitis in humans. A study which looked at the BBB permeability of the pathogen reported that virulence is because of a newly identified Fic domain-containing protein, BifA. BifA was required for SEZ to cross the BBB and to cause meningitis in mice model as well as translocation across HBMEC monolayers. BifA activation of moesin appears to constitute a key mechanism by which SEZ disrupts endothelial monolayer integrity to penetrate the BBB [126]. Streptococcus pneumoniae causes bacterial meningitis. PLY (pneumolysin), a cytotoxin from pneumococcus, is related to the infection across BBB. PLY leads to the high expression of CERB-binding protein (CBP), which can lead to releasing of tumor necrosis factor $\alpha$ thereby enhancing apoptosis of cells, a significant factor leading to permeabilization of BBB [127].

Microglia has an important role in pathophysiology of bacterial meningitis. Microglia is responsible in triggering neuroinflammation, characterized by chemokine and cytokine release leading to white blood cell trafficking through vascular endothelium of brain and BBB disruption [128]. 
Ureaplasma species (spp.) are known to cause invasive diseases in immunocompromised adults and in neonates, including neonatal meningitis. Ureaplasma spp. may provoke barrier breakdown by inducing apoptosis in HBMEC [129].

\section{Discussion}

This research article has given a molecular and cellular level understanding of BBB functions and has raised awareness about the role of BBB dysfunction in the pathogenesis of different CNS diseases mentioned above. our understanding of the BBB at the molecular and cellular level will continue to grow based on findings in rodent models, the question remains to what extent these findings are translatable to human BBB. Elucidating species- specific similarities and differences in endothelial and pericyte expression profiles remains to be fully determined at the transcriptome and proteome levels. In terms of studying BBB function in humans, recent neuroimaging studies made important advances to allow us to measure regional BBB integrity and quantify subtle changes in BBB permeability in CNS regions as small as hippocampal CA1, CA3, and dentate gyrus subfields which was lacking previously [126].

A critical question to ask and think moving forward is to understand what aspects of this BBB dysfunction are healing and what aspects are pathological. The current thinking should be at what inflammation level in brain or blood can cause BBB to become dysfunctional, leaky or even damaged completely and how to protect or repair a broken BBB. Thinking should also focus on delivering drugs into CNS which can cross BBB without causing any disturbance in function. We expect that imaging methods with 7T MRI and new technologies will improve our ability to detect BBB changes in humans, and determine how they relate to blood flow changes, changes in structural and functional brain connectivity, and cognitive and motor deficits in different neurodegenerative disorders, as well as cell-specific biomarkers of the vascular injury and NVU in biofluids.

\section{Conclusion}

BBB is an important cellular and molecular barrier that tightly controls the mileu of CNS to allow for proper neuronal functioning. This restrictive barrier is an extremely important because it acts as a gatekeeper to protect the CNS from toxins, pathogens, inflammation which can lead to pathology and disease as well as development of CNSacting therapeutics which can safely pass through it without causing any disintegrity. Finally, based on the current state of our knowledge, it is probably time to think about BBB not only as an impermeable cellular membrane which protects brain from peripheral influences and should be breached for therapeutic CNS drug delivery, but also as an enormous source of understudied molecular and cellular targets in the disease state, which if explored could change the way we think about brain diseases and could lead to development of important new BBB-based approaches to treat them. Further studies are required in future.

\section{Acknowledgement}

The author has no relevant affiliations or financial involvement with any organization or entity with a financial interest in or financial conflict with the subject matter or materials discussed in the manuscript. Thanks to my mentor Prof. Hui Fang Shang for constant support and Dr Swati for revising the drafted manuscript.

\section{References}

1. Zlokovic BV. The blood-brain barrier in health and chronic neurodegenerative disorders. Neuron. 2018; 57: 178-201. PubMed: https://pubmed.ncbi.nlm.nih.gov/18215617/

2. Daneman R. The blood-brain barrier in health and disease. Ann Neurol. 2018; 72: 648-672. PubMed https://www.ncbi.nlm.nih.gov/ pmc/articles/PMC4292164/

3. Montagne A, Zhao Z, Zlokovic BV. Alzheimer's disease: a matter of blood-brain barrier dysfunction? J Exp Med. 2017; 214: 3151-3169. PubMed: https://pubmed.ncbi.nlm.nih.gov/29061693/

4. Dong X. Current Strategies for Brain Drug Delivery. Theranostics. 2018; 8: 1481-1493.

PubMed: https://www.ncbi.nlm.nih.gov/pmc/articles/PMC5858162/

5. Iadecola C. The pathobiology of vascular dementia. Neuron. 2013; 80: 844-866.

PubMed: https://pubmed.ncbi.nlm.nih.gov/24267647/

6. ladecola C. The Neurovascular Unit Coming of Age: A Journey through NeurovascularCoupling in Health and Disease. Neuron. 2017;96:17-42. PubMed: https://pubmed.ncbi.nlm.nih.gov/28957666/

7. Kisler K, Nelson AR, Montagne A, Zlokovic BV. Cerebral blood flow regulation and neurovascular dysfunction in Alzheimer disease. Nat Rev Neurosci. 2017; 18: 419-434.

PubMed: https://pubmed.ncbi.nlm.nih.gov/28515434/

8. Zhao Z, Nelson AR, Betsholtz C, Zlokovic BV. Establishment and Dysfunction of the Blood-Brain Barrier. Cell. 2015; 163: 1064-1078. PubMed: https://pubmed.ncbi.nlm.nih.gov/26590417/

9. Thurgur H, Pinteaux E. Microglia in the Neurovascular Unit: BloodBrain Barrier-microglia Interactions After Central Nervous System Disorders. Neuroscience. 2018; 405 :55-67.

PubMed: https://pubmed.ncbi.nlm.nih.gov/31007172

10. Hawkins BT. The Blood-Brain Barrier/Neurovascular Unit in Health and Disease. Pharmacological Rev. 2005; 57: 173-185. PubMed: https://www.ncbi.nlm.nih.gov/pmc/articles/PMC3757302/

11. Sweeney, Melanie D, Zlokovic BV. Pericytes of the neurovascular unit: key functions and signaling pathways. Nat Neurosci. 2005; 19: 771-783. PubMed: https://pubmed.ncbi.nlm.nih.gov/27227366/

12. Zlokovic BV. Neurovascular pathways to neurodegeneration in Alzheimer's disease and other disorders. Nat Rev Neurosci. 2011; 12 : 723-738.

PubMed: https://pubmed.ncbi.nlm.nih.gov/22048062/

13. Mancuso MR, Kuhnert F, Kuo CJ. Developmental angiogenesis of the central nervous system. Lymphat Res Biol. 2008; 6: 173-180. PubMed: https://www.ncbi.nlm.nih.gov/pmc/articles/PMC2712664/

14. Posokhova E, Shukla A, Seaman S, Volate S, Hilton MB, et al. GPR124 functions as a WNT7-specific coactivator of canonical-catenin signaling. Cell Reports. 2015; 10: 123-130.

15. Alvarez JI, Dodelet-Devillers A, Kebir H, Ifergan I, Fabre PJ, et al. The 
Hedgehog pathway promotes blood-brain barrier integrity and CNS immune quiescence. Science. 2011; 334: 1727-1731.

PubMed: https://pubmed.ncbi.nlm.nih.gov/22144466/

16. Armulik A, Genové G, Betsholtz C. Pericytes: developmental, physiological, and pathological perspectives, problems, and promises. Dev Cell. 2011; 21: 193-215.

PubMed: https://pubmed.ncbi.nlm.nih.gov/21839917/

17. Le Guelte A, Galan-Moya EM, Dwyer J, Treps L, Kettler G, et al. Semaphorin 3A elevates endothelial cell permeability through PP2A inactivation. J Cell Sci. 2012; 125: 4137-4146.

PubMed: https://pubmed.ncbi.nlm.nih.gov/22685328/

18. Blanchette M, Daneman R. Formation and maintenance of the BBB. Mechanisms of Development. 2015; 138, 8-16.

PubMed: https://pubmed.ncbi.nlm.nih.gov/26215350/

19. Obermeier B, Daneman R, Ransohoff RM. Development, maintenance and disruption of the blood-brain barrier. Nat Med. 2013; 19: 1584-1596. PubMed: https://www.ncbi.nlm.nih.gov/pmc/articles/PMC4080800/

20. Sun M, Ma K, Wen J, Wang G, Zhang C, et al. A Review of the Brain-Gut-Microbiome Axis and the Potential Role of Microbiota in Alzheimer's Disease. J Alzheimers Dis. 73: 849-865.

PubMed: https://pubmed.ncbi.nlm.nih.gov/31884474/

21. Erickson MA, Banks WA. Blood-brain barrier dysfunction as a cause and consequence of Alzheimer's disease. J Cereb Blood Flow Metab 2013; 33: 1500-1513

PubMed: https://pubmed.ncbi.nlm.nih.gov/23921899/

22. Deane R, Bell RD, Sagare A, Zlokovic BV. Clearance of amyloid-beta peptide across the blood-brain barrier: implication for therapies in Alzheimer's disease. CNS Neurol Disord Drug Targets. 2009; 8: 16-30. PubMed: https://pubmed.ncbi.nlm.nih.gov/19275634/

23. Yamada K, Hashimoto T, Yabuki C, Nagae Y, Tachikawa M, et al. The low density lipoprotein receptor-related protein 1 mediates uptake of amyloid beta peptides in an in vitro model of the blood-brain barrier cells. J Biol Chem. 2008; 283: 34554-34562.

PubMed: https://pubmed.ncbi.nlm.nih.gov/18940800/

24. Ujiie M, Dickstein DL, Carlow DA, Jefferies WA. Blood-brain barrier permeability precedes senile plaque formation in an Alzheimer disease model. Microcirculation. 2003; 10: 463-470.

PubMed: https://pubmed.ncbi.nlm.nih.gov/14745459/

25. Wu S, Liu H, Zhao H, Wang $\mathrm{X}$, Chen J, et al. Environmental lead exposure aggravates the progression of Alzheimer's disease in mice by targeting on blood brain barrier. Toxicol Lett. 2020; 319: 138-147. PubMed: https://pubmed.ncbi.nlm.nih.gov/31730887/

26. Shin Y, Choi SH, Kim E, Bylykbashi E, Kim JA, et al. Blood-Brain Barrier Dysfunction in a 3D In Vitro Model of Alzheimer's Disease. Adv Sci. 2019; 6: 1900962.

PubMed: https://www.ncbi.nlm.nih.gov/pmc/articles/PMC6794630/

27. Miners JS, Kehoe PG, Love S, Zetterberg H, Blennow K. CSF evidence of pericyte damage in Alzheimer's disease is associated with markers of blood-brain barrier dysfunction and disease pathology. Alzheimers Res Ther. 2019; 11: 81

PubMed: https://pubmed.ncbi.nlm.nih.gov/31521199/

28. Le Stunff H, Véret J, Kassis N, Denom J, Meneyrol K, et al. Deciphering the Link Between Hyperhomocysteinemia and Ceramide Metabolism in Alzheimer-Type Neurodegeneration. Front Neurol. 2019; 10; 807. PubMed: https://www.ncbi.nlm.nih.gov/pmc/articles/PMC6684947/

29. He JT, Zhao X, Xu L, Mao CY. Vascular Risk Factors and Alzheimer's Disease: Blood-Brain Barrier Disruption, Metabolic Syndromes, and Molecular Links. J Alzheimers Dis. 2020; 73: 39-58.

PubMed: https://pubmed.ncbi.nlm.nih.gov/31815697/

30. Rivera S, García-González L, Khrestchatisky M, Baranger K Metalloproteinases and their tissue inhibitors in Alzheimer's disease and other neurodegenerative disorders. Cell Molecular Life Sci. 2019; 76: 3167-3191.

PubMed: https://pubmed.ncbi.nlm.nih.gov/31197405/
31. Ozkizilcik A, Sharma A, Lafuente JV, Muresanu DF, Castellani RJ, et al. Nanodelivery of cerebrolysin reduces pathophysiology of Parkinson's disease. Progress Brain Res. 2019; 201-246. PubMed: https://pubmed.ncbi.nlm.nih.gov/30961868/

32. Matsumoto J, Stewart T, Sheng L, Li N, Bullock k, et al. Transmission of $\alpha$-synuclein-containing erythrocyte-derived extracellular vesicles across the blood-brain barrier via adsorptive mediated transcytosis: another mechanism for initiation and progression of Parkinson's disease?. Acta Neuropathol Commun. 2017; 5: 71. PubMed: https://pubmed.ncbi.nlm.nih.gov/28903781/

33. Olmedo-Díaz S, Estévez-Silva H, Orädd G, Af Bjerkén S, Marcellino $D$, et al. An altered blood-brain barrier contributes to brain iron accumulation and neuroinflammation in the 6-OHDA rat model of Parkinson's disease. Neuroscience. 2017; 362: 141-151.

PubMed: https://pubmed.ncbi.nlm.nih.gov/28842186/

34. Gray MT, Woulfe JM. Striatal blood-brain barrier permeability in Parkinson's disease. J Cereb Blood Flow Metab. 2015; 35: 747-750. PubMed: https://pubmed.ncbi.nlm.nih.gov/25757748/

35. Dohgu S, Takata F, Matsumoto J, Kimura I, Yamauchi A, et al Monomeric a-synuclein induces blood-brain barrier dysfunction through activated brain pericytes releasing inflammatory mediators in vitro. Microvascular Res. 2019. 124: 61-66.

PubMed: https://pubmed.ncbi.nlm.nih.gov/30885616/

36. Chung YC, Kim YS, Bok E, Yune TY, Maeng S, et al. MMP-3 contributes to nigrostriatal dopaminergic neuronal loss, BBB damage, and neuroinflammation in an MPTP mouse model of Parkinson's disease. Mediat Inflamm. 2013: 370526.

37. Lerner RP, Francardo V, Fujita K, Bimpisidis Z, Jourdain VA, et al. Levodopa-induced abnormal involuntary movements correlate with altered permeability of the blood-brain-barrier in the basal ganglia. Sci Rep. 2017; 7: 16005.

PubMed: https://pubmed.ncbi.nlm.nih.gov/29167476/

38. Ohlin KE, Sebastianutto I, Adkins CE, Lundblad C, Lockman PR, et al. Impact of L-DOPA treatment on regional cerebral blood flow and metabolism in the basal ganglia in a rat model of Parkinson's disease. Neuroimage. 2012; 61: 228-239.

PubMed: https://pubmed.ncbi.nlm.nih.gov/22406356/

39. Duran-Vilaregut J, del Valle J, Manich G, Camins A, Pallas M, et al Role of matrix metalloproteinase- 9(MMP-9) in striatal blood-brain barrier disruption in a 3-nitropropionic acid model of Huntington's disease. Neuropathol Appl Neuro. 2011; 37: 525-537. PubMed: https://pubmed.ncbi.nlm.nih.gov/21175737/

40. Drouin-Ouellet J, Sawiak SJ, Cisbani G, Lagacé M, Kuan W, et al. Cerebrovascular and blood-brain barrier impairments in Huntington's disease: potential implications for its pathophysiology. Ann Neurol. 2015; 78: 160-177.

PubMed: https://pubmed.ncbi.nlm.nih.gov/25866151/

41. Hsiao HY, Chen YC, Huang $\mathrm{CH}$, Chen CC, Hsu Y, et al. Aberrant astrocytes impair vascular reactivity in Huntington disease. Ann Neurol. 2015; 78: 178-192.

PubMed: https://pubmed.ncbi.nlm.nih.gov/25914140/

42. Lin $\mathrm{CY}$, Hsu YH, Lin MH, Yang TH, Chen HM, et al. Neurovascular abnormalities in humans and mice with Huntington's disease. Exp Neurol. 2013; 250: 20-30.

PubMed: https://pubmed.ncbi.nlm.nih.gov/24036415/

43. Lim RG, Quan C, Reyes-Ortiz AM, et al. Huntington's Disease iPSCDerived Brain Microvascular Endothelial Cells Reveal WNT-Mediated Angiogenic and Blood-Brain Barrier Deficits. Cell Rep. 2017; 19: 13651377.

PubMed: https://pubmed.ncbi.nlm.nih.gov/28514657/

44. Di Pardo A, Amico E, Scalabrì $F$, et al. Impairment of blood-brain barrier is an early event in R6/2 mouse model of Huntington Disease. Sci Rep. 2017; 7: 41316.

PubMed: https://www.ncbi.nlm.nih.gov/pmc/articles/PMC5259798/ 
45. Garbuzova-Davis S, Haller E, Saporta S, Kolomey I, Nicosia SV, et al. Ultrastructure of blood-brain barrier and blood-spinal cord barrier in SOD1 mice modeling ALS. Brain Res. 2007; 1157: 126-137. PubMed: https://pubmed.ncbi.nlm.nih.gov/17512910/

46. Miyazaki K, Ohta $\mathrm{Y}$, Nagai M, et al. Disruption of neurovascular unit prior to motor neuron degeneration in amyotrophic lateral sclerosis. J Neurosci Res. 2011; 89: 718-728.

PubMed: https://pubmed.ncbi.nlm.nih.gov/21337372/

47. Winkler EA, Sengillo JD, Sagare AP, et al. Blood-spinal cord barrier disruption contributes to early motor-neuron degeneration in ALSmodel mice. Proc Natl Acad Sci U S A. 2014; 111: E1035-E1042. PubMed: https://pubmed.ncbi.nlm.nih.gov/24591593/

48. Zhong Z, llieva H, Hallagan L, et al. Activated protein $\mathrm{C}$ therapy slows ALS-like disease in mice by transcriptionally inhibiting SOD1 in motor neurons and microglia cells. J Clin Invest. 2009; 119: 3437-3449. PubMed: https://www.ncbi.nlm.nih.gov/pmc/articles/PMC2769191/

49. Zhong Z, Deane R, Ali Z, et al. ALS-causing SOD1 mutants generate vascular changes prior to motor neuron degeneration. Nat Neurosci. 2008; 11: 420-422.

PubMed: https://pubmed.ncbi.nlm.nih.gov/18344992/

50. Nicaise $\mathrm{C}$, Mitrecic D, Demetter $\mathrm{P}$, et al. Impaired blood-brain and blood-spinal cord barriers in mutant SOD1-linked ALS rat. Brain Res. 2009; 1301: 152-162.

PubMed: https://pubmed.ncbi.nlm.nih.gov/19748495/

51. Evans MC, Serres S, Khrapitchev AA, Stolp HB, Anthony DC, et al. $\mathrm{T}_{2}$-weighted MRI detects presymptomatic pathology in the SOD1 mouse model of ALS. J Cereb Blood Flow Metab. 2014; 34: 785-793. PubMed: https://www.ncbi.nlm.nih.gov/pmc/articles/PMC4013759/

52. Andjus PR, Bataveljić D, Vanhoutte G, Mitrecic D, Pizzolante F, et al. In vivo morphological changes in animal models of amyotrophic lateral sclerosis and Alzheimer's-like disease: MRI approach. Anat Rec (Hoboken). 2009; 292: 1882-1892.

PubMed: https://pubmed.ncbi.nlm.nih.gov/19943341/

53. Bataveljić D, Nikolić L, Milosević M, Todorović N, Andjus PR. Changes in the astrocytic aquaporin-4 and inwardly rectifying potassium channel expression in the brain of the amyotrophic lateral sclerosis SOD1(G93A) rat model. Glia. 2012; 60: 1991-2003.

PubMed: https://pubmed.ncbi.nlm.nih.gov/22987392/

54. Bataveljić D, Stamenković S, Bačić G, Andjus PR. Imaging cellular markers of neuroinflammation in the brain of the rat model of amyotrophic lateral sclerosis. Acta Physiol Hung. 2011; 98: 27-31. PubMed: https://pubmed.ncbi.nlm.nih.gov/21388928/

55. Garbuzova-Davis S, Saporta S, Haller E, et al. Evidence of compromised blood-spinal cord barrier in early and late symptomatic SOD1 mice modeling ALS. PLoS One. 2007; 2: e1205.

PubMed: https://pubmed.ncbi.nlm.nih.gov/18030339/

56. Nicaise C, Soyfoo MS, Authelet M, et al. Aquaporin-4 overexpression in rat ALS model. Anat Rec (Hoboken). 2009; 292: 207-213.

PubMed: https://www.ncbi.nlm.nih.gov/pmc/articles/PMC5562093/

57. Garbuzova-Davis S, Hernandez-Ontiveros DG, Rodrigues MC, et al. Impaired blood-brain/spinal cord barrier in ALS patients. Brain Res. 2012; 1469: 114-128.

PubMed: https://pubmed.ncbi.nlm.nih.gov/22750125/

58. Wu Y, Yang X, Li X, Wang H, Wang T. Elevated cerebrospinal fluid homocysteine is associated with blood-brain barrier disruption in amyotrophic lateral sclerosis patients Neurol Sci. 2020; 41: 1865-1872. PubMed: https://pubmed.ncbi.nlm.nih.gov/32086685/

59. Wang $Y$, Jin $S$, Sonobe $Y$, et al. Interleukin-1 $\beta$ induces blood-brain barrier disruption by downregulating Sonic hedgehog in astrocytes. PLoS One. 2014; 9: e110024.

PubMed: https://pubmed.ncbi.nlm.nih.gov/25313834/

60. Ticozzi N, Tiloca $\mathrm{C}$, Mencacci NE, et al. Oligoclonal bands in the cerebrospinal fluid of amyotrophic lateral sclerosis patients with disease-associated mutations. J Neurol. 2013; 260: 85-92. PubMed: https://www.ncbi.nlm.nih.gov/pmc/articles/PMC4196642/

61. Henkel JS, Beers DR, Wen S, Bowser R, Appel SH. Decreased mRNA expression of tight junction proteins in lumbar spinal cords of patients with ALS. Neurology. 2009; 72: 1614-1616.

PubMed: https://pubmed.ncbi.nlm.nih.gov/19414730/

62. Garbuzova-Davis S, Woods RL 3rd, Louis MK, et al. Reduction of circulating endothelial cells in peripheral blood of ALS patients. PLoS One. 2010; 5: e10614.

PubMed: https://pubmed.ncbi.nlm.nih.gov/20485543/

63. Ortiz GG, Pacheco-Moisés FP, Macías-Islas MÁ, Flores-Alvarado LJ, Mireles-Ramírez MA, et al. Role of the blood-brain barrier in multiple sclerosis. Arch Med Res. 2014; 45: 687-697.

PubMed: https://pubmed.ncbi.nlm.nih.gov/25431839/

64. Alvarez JI, Cayrol R, Prat A. Disruption of central nervous system barriers in multiple sclerosis. Biochim Biophys Acta. 2011; 1812: 252-264. PubMed: https://pubmed.ncbi.nlm.nih.gov/20619340/

65. Setiadi AF, Abbas AR, Jeet S, Wong K, Bischof A, et al. IL-17A is associated with the breakdown of the blood-brain barrier in relapsingremitting multiple sclerosis. J Neuroimmunol. 2019; 332: 147-154. PubMed: https://pubmed.ncbi.nlm.nih.gov/31034962/

66. Niu J, Tsai HH, Hoi KK, Huang N, Yu G, et al. Aberrant oligodendroglialvascular interactions disrupt the blood-brain barrier, triggering CNS inflammation. Nat Neurosci. 2019; 22: 709-718. PubMed: https://pubmed.ncbi.nlm.nih.gov/30988524/

67. Haarmann A, Schuhmann M, Silwedel C, Monoranu CM, Stoll G, et al. Human Brain Endothelial CXCR2 is Inflammation-Inducible and Mediates CXCL5- and CXCL8-Triggered Paraendothelial Barrier Breakdown. Int J Molecular Sci. 2019; 20: 602.

PubMed: https://www.ncbi.nlm.nih.gov/pmc/articles/PMC6387364/

68. Greene C, Hanley N, Campbell M. Claudin-5: gatekeeper of neurological function. Fluids and Barriers of the CNS. 2019; 16: 3. PubMed: https://pubmed.ncbi.nlm.nih.gov/30691500/

69. Uchida Y, Sumiya T, Tachikawa M, et al. Involvement of Claudin-11 in Disruption of Blood-Brain, -Spinal Cord, and -Arachnoid Barriers in Multiple Sclerosis. Mol Neurobiol. 2019; 56: 2039-2056.

70. Eisele $\mathrm{P}$, Konstandin S, Szabo K, Ebert A, Roßmanith $\mathrm{C}$, et al Temporal evolution of acute multiple sclerosis lesions on serial sodium (23Na) MRI. Mult Scler Relat Disord. 2019; 29: 48-54. PubMed: https://pubmed.ncbi.nlm.nih.gov/30669020/

71. Filippi M, Rocca MA, Martino G, Horsfield MA, Comi G. Magnetization transfer changes in the normal appearing white matter precede the appearance of enhancing lesions in patients with multiple sclerosis. Ann Neurol. 1998; 43: 809-814.

72. Vos CM, Geurts JJ, Montagne L, et al. Blood-brain barrier alterations in both focal and diffuse abnormalities on postmortem MRI in multiple sclerosis. Neurobiol Dis. 2005; 20: 953-960.

PubMed: https://pubmed.ncbi.nlm.nih.gov/16039866/

73. Cramer SP, Modvig S, Simonsen HJ, Frederiksen JL, Larsson HB. Permeability of the blood-brain barrier predicts conversion from optic neuritis to multiple sclerosis. Brain. 2015; 138: 2571-2583.

PubMed: https://pubmed.ncbi.nlm.nih.gov/26187333/

74. Sheikh MH, Henson SM, Loiola RA, Mercurio S, Colamatteo A, et al Immuno-metabolic impact of the multiple sclerosis patients' sera on endothelial cells of the blood-brain barrier. J Neuroinflammation. 2020; 17: 153.

PubMed: https://pubmed.ncbi.nlm.nih.gov/32386505/

75. Wang S, Millward JM, Hanke-Vela L, et al. MR Elastography-Based Assessment of Matrix Remodeling at Lesion Sites Associated With Clinical Severity in a Model of Multiple Sclerosis. Front Neurol. 2020; 10: 1382.

PubMed: https://www.ncbi.nlm.nih.gov/pmc/articles/PMC6970413/ 
76. Sacktor N, Skolasky RL, Seaberg E, Munro C, Becker JT, et al. Prevalence of HIV-associated neurocognitive disorders in the Multicenter AIDS Cohort Study. Neurology. 2016; 86: 334-340. PubMed: https://pubmed.ncbi.nlm.nih.gov/26718568/

77. Jessen Krut J, Mellberg T, Price RW, Hagberg L, Fuchs D, et al. Biomarker evidence of axonal injury in neuroasymptomatic HIV-1 patients. PLoS One. 2014; 9: e88591.

PubMed: https://pubmed.ncbi.nlm.nih.gov/24523921/

78. Xing Y, Shepherd N, Lan J, Li W, Rane S, et al. MMPs/TIMPs imbalances in the peripheral blood and cerebrospinal fluid are associated with the pathogenesis of HIV-1-associated neurocognitive disorders. Brain Behavior Immunity. 2017; 65: 161-172.

PubMed: https://pubmed.ncbi.nlm.nih.gov/28487203/

79. Strazza M, Pirrone V, Wigdahl B, Nonnemacher MR. Breaking down the barrier: the effects of HIV-1 on the blood-brain barrier. Brain Res. 2011; 1399: 96-115.

PubMed:. https://www.ncbi.nlm.nih.gov/pmc/articles/PMC3139430/

80. Niu F, Yao H, Zhang W, Sutliff RL, Buch S. Tat 101-mediated enhancement of brain pericyte migration involves platelet-derived growth factor subunit B homodimer: implications for human immunodeficiency virus-associated neurocognitive disorders. J Neurosci. 2014; 34: 11812-11825.

PubMed: https://www.ncbi.nlm.nih.gov/pmc/articles/PMC4145180/

81. Doherty CP, O'Keefe E, Wallace E, Loftus T, Keaney J, et al. BloodBrain Barrier Dysfunction as a Hallmark Pathology in Chronic Traumatic Encephalopathy. J Neuropathol Exp Neurol. 2016; 75: 656-662. PubMed: https://www.ncbi.nlm.nih.gov/pmc/articles/PMC4913433/

82. Farrell M, Aherne S, O'Riordan S, O'Keeffe E, Greene C, Campbell M. Blood-brain barrier dysfunction in a boxer with chronic traumatic encephalopathy and schizophrenia. Clin Neuropathol. 2019; 38: 51-58. PubMed: https://pubmed.ncbi.nlm.nih.gov/30574863/

83. Glushakova OY, Glushakov AO, Borlongan CV, Valadka AB, Hayes RL, et al. Role of Caspase-3-Mediated Apoptosis in Chronic Caspase-3Cleaved Tau Accumulation and Blood-Brain Barrier Damage in the Corpus Callosum after Traumatic Brain Injury in Rats. J Neurotrauma. 2018; 35: 157-173.

84. Demock M, Kornguth S. A Mechanism for the Development of Chronic Traumatic Encephalopathy From Persistent Traumatic Brain Injury. J Exp Neurosci. 2019; 13: 1179069519849935.

PubMed: https://www.ncbi.nlm.nih.gov/pmc/articles/PMC6537483/

85. Glushakova OY, Johnson D, Hayes RL. Delayed increases in microvascular pathology after experimental traumatic brain injury are associated with prolonged inflammation, blood-brain barrier disruption, and progressive white matter damage. J Neurotrauma. 2014; 31: 11801193.

PubMed: https://pubmed.ncbi.nlm.nih.gov/24564198

86. Keep RF, Xiang J, Ennis SR, Andjelkovic A, Hua Y, et al. Blood-brain barrier function in intracerebral hemorrhage. Acta Neurochir Suppl (Wien). 2008; 105: 73-77.

PubMed: https://pubmed.ncbi.nlm.nih.gov/19066086/

87. Khatri R, McKinney AM, Swenson B, Janardhan V. Blood-brain barrier, reperfusion injury, and hemorrhagic transformation in acute ischemic stroke. Neurology. 2012; 79: S52-S57.

PubMed: https://pubmed.ncbi.nlm.nih.gov/23008413/

88. Prakash R, Carmichael ST. Blood-brain barrier breakdown and neovascularization processes after stroke and traumatic brain injury. Curr Opin Neurol. 2015; 28: 556-564.

PubMed: https://www.ncbi.nlm.nih.gov/pmc/articles/PMC5267616/

89. Keaney J, Campbell M. The dynamic blood-brain barrier. FEBS J. 2015; 282: 4067-4079.

PubMed: https://pubmed.ncbi.nlm.nih.gov/26277326/

90. The glucagon-like peptide- 1 receptor agonist reduces inflammation and blood-brain barrier breakdown in an astrocyte-dependent manner in experimental stroke. J Neuroinflammation. 2019; 16: 242.

PubMed: https://pubmed.ncbi.nlm.nih.gov/31779652/

91. Sarvari S, Moakedi F, Hone E, Simpkins JW, Ren X. Mechanisms in blood-brain barrier opening and metabolism-challenged cerebrovascular ischemia with emphasis on ischemic stroke. Metab Brain Dis. 2020; 35: 851-868

PubMed: https://pubmed.ncbi.nlm.nih.gov/32297170/

92. Guo $F, X u D$, Lin $Y$, et al. Chemokine CCL2 contributes to BBB disruption via the p38 MAPK signaling pathway following acute intracerebral hemorrhage. FASEB J. 2020; 34: 1872-1884.

PubMed: https://pubmed.ncbi.nlm.nih.gov/31914700/

93. Li Y, Wu P, Bihl JC, Shi H. Underlying Mechanisms and Potential Therapeutic Molecular Targets in Blood-Brain Barrier Disruption after SubarachnoidHemorrhage. CurrNeuropharmacol.2020;18:1168-1179. PubMed: https://pubmed.ncbi.nlm.nih.gov/31903882/

94. Victoria ECG, Toscano ECB, Oliveira FMS, et al. Up-regulation of brain cytokines and metalloproteinases 1 and 2 contributes to neurological deficit and brain damage in transient ischemic stroke. Microvasc Res. 2020; 129: 103973.

PubMed: https://pubmed.ncbi.nlm.nih.gov/31891716/

95. Bas,kaya MK, Rao AM, Dog an A, Donaldson D, Dempsey RJ. The biphasic opening of the blood-brain barrier in the cortex and hippocampus after traumatic brain injury in rats. Neurosci Lett. 1997; 226: 33-36.

PubMed: https://pubmed.ncbi.nlm.nih.gov/9153635/

96. Habgood MD, Bye N, Dziegielewska KM, Ek CJ, Lane MA, et al Changes in blood-brain barrier permeability to large and smal molecules following traumatic brain injury in mice. Eur $\mathrm{J}$ Neurosci. 2007; 25: 231-238.

PubMed: https://pubmed.ncbi.nlm.nih.gov/17241284/

97. Kawoos U, Abutarboush R, Zarriello S, Qadri A, Ahlers ST, et al. $\mathrm{N}$-acetylcysteine Amide Ameliorates Blast-Induced Changes in BloodBrain Barrier Integrity in Rats. Front Neurol. 2019; 10: 650.

PubMed: https://www.ncbi.nlm.nih.gov/pmc/articles/PMC6607624/

98. Szarka N, Toth L, Czigler A, Kellermayer Z, Ungvari Z, et al. Single Mild Traumatic Brain Injury Induces Persistent Disruption of the Blood-Brain Barrier, Neuroinflammation and Cognitive Decline in Hypertensive Rats. Int J Molecular Sci. 2019; 20: 3223.

PubMed: https://pubmed.ncbi.nlm.nih.gov/31262044/

99. Kuriakose M, Younger D, Ravula AR, Alay E, Rama Rao KV, et al Synergistic Role of Oxidative Stress and Blood-Brain Barrier Permeability as Injury Mechanisms in the Acute Pathophysiology of Blast-induced Neurotrauma. Sci Rep. 2019; 9: 7717.

100. Michinaga S, Koyama Y. Dual Roles of Astrocyte-Derived Factors in Regulation of Blood-Brain Barrier Function after Brain Damage. Int J Molecular Sci. 2019; 20: 571.

PubMed: https://www.ncbi.nlm.nih.gov/pmc/articles/PMC6387062/

101. Price L, Wilson C, Grant G. Frontiers in neuroscience bloodbrain barrier pathophysiology following traumatic brain injury. In Translational Research in Traumatic Brain Injury; Laskowitz, D, Grant, G, Eds.; CRC Press/Taylor and Francis Group@: Boca Raton, FL, USA, 2016.

PubMed: https://pubmed.ncbi.nlm.nih.gov/26583184/

102. Hay JR, Johnson VE, Young AM, Smith DH, Stewart W. Blood-Brain Barrier Disruption Is an Early Event That May Persist for Many Years After Traumatic Brain Injury in Humans. J Neuropathol Exp Neurol. 2015; 74: 1147-1157.

PubMed: https://pubmed.ncbi.nlm.nih.gov/26574669/

103. Johnson VE, Weber MT, Xiao R, et al. Mechanical disruption of the blood-brain barrier following experimental concussion. Acta Neuropathol. 2018; 135: 711-726.

PubMed: https://www.ncbi.nlm.nih.gov/pmc/articles/PMC6532777/

104. Hagos FT, Empey PE, Wang P, et al. Exploratory Application of Neuropharmacometabolomics in Severe Childhood Traumatic Brain 
Injury. Crit Care Med. 2018; 46: 1471-1479.

PubMed: https://pubmed.ncbi.nlm.nih.gov/29742587/

105. Higashida T, Kreipke CW, Rafols JA, et al. The role of hypoxiainducible factor-1 $\alpha$, aquaporin-4, and matrix metalloproteinase-9 in blood-brain barrier disruption and brain edema after traumatic brain injury. J Neurosurg. 2011; 114: 92-101.

PubMed: https://pubmed.ncbi.nlm.nih.gov/20617879/

106. Pan R, Yu K, Weatherwax T, Zheng H, Liu W, et al. Blood Occludin Level as a Potential Biomarker for Early Blood Brain Barrier Damage Following Ischemic Stroke. Sci Rep. 2017; 7: 40331.

PubMed: https://www.ncbi.nlm.nih.gov/pmc/articles/PMC5228160/

107. Zongo D, Ribéreau-Gayon R, Masson F, et al. S100-B protein as a screening tool for the early assessment of minor head injury. Ann Emerg Med. 2012; 59: 209-218

PubMed: https://pubmed.ncbi.nlm.nih.gov/21944878/

108. Neher MD, Keene CN, Rich MC, Moore HB, Stahel PF. Serum biomarkers for traumatic brain injury. South Med J. 2014; 107: 248-255. PubMed: https://www.ncbi.nlm.nih.gov/pmc/articles/PMC4774463/

109. Pham N, Akonasu H, Shishkin R, Taghibiglou C. Plasma soluble prion protein, a potential biomarker for sport-related concussions: a pilot study. PLoS One. 2015; 10: e0117286.

PubMed: https://www.ncbi.nlm.nih.gov/pmc/articles/PMC4314282/

110. Rigau V, Morin $M$, Rousset $M C$, de Bock $F$, Lebrun $A$, et al Angiogenesis is associated with blood-brain barrier permeability in temporal lobe epilepsy. Brain. 2007; 130: 1942-1956. PubMed: https://pubmed.ncbi.nlm.nih.gov/17533168/

111. Swissa E, Serlin Y, Vazana U, Prager O, Friedman A. Blood-brain barrier dysfunction in status epileptics: Mechanisms and role in epileptogenesis. Epilepsy Behav. 2019; 101: 106285.

PubMed: https://www.ncbi.nlm.nih.gov/pmc/articles/PMC4842020/

112. Kim SY, Buckwalter M, Soreq H, Vezzani A, Kaufer D. Blood-brain barrier dysfunction-induced inflammatory signaling in brain pathology and epileptogenesis. Epilepsia. 2012; 53: 37-44.

PubMed: https://pubmed.ncbi.nlm.nih.gov/23134494/

113. Giza CC, Hovda DA. The new neurometabolic cascade of concussion. Neurosurgery. 2014; 75: S24-S33.

PubMed: https://pubmed.ncbi.nlm.nih.gov/25232881

114. Vezzani A, French J, Bartfai T, Baram TZ. The role of inflammation in epilepsy. Nat Rev Neurol. 2011; 7: 31-40.

PubMed: https://pubmed.ncbi.nlm.nih.gov/21135885

115. van Vliet EA, da Costa Araújo S, Redeker S, van Schaik R, Aronica E, et al. Blood-brain barrier leakage may lead to progression of temporal lobe epilepsy. Brain. 2007; 130: 521-534.

PubMed: https://pubmed.ncbi.nlm.nih.gov/17124188/

116. Van Vliet EA, Aronica E, Gorter JA. Role of blood-brain barrier in temporal lobe epilepsy and pharmacoresistance. Neuroscience. 2017; 277: 455-473.

PubMed: https://pubmed.ncbi.nlm.nih.gov/25080160/

117. Liu XX, Yang L, Shao LX, et al. Endothelial Cdk5 deficit leads to the development of spontaneous epilepsy through CXCL1/CXCR2mediated reactive astrogliosis. J Exp Med. 2020; 217: e20180992. PubMed: https://pubmed.ncbi.nlm.nih.gov/31699822/
118. Dadas A, Janigro D. Breakdown of blood brain barrier as a mechanism of post-traumatic epilepsy. Neurobiol Dis. 2019; 123: 20-26. PubMed: https://pubmed.ncbi.nlm.nih.gov/30030025/

119. Gorter JA, Aronica E, van Vliet EA. The Roof is Leaking and a Storm is Raging: Repairing the Blood-Brain Barrier in the Fight Against Epilepsy. Epilepsy Currents. 2019; 19: 177-181.

PubMed: https://pubmed.ncbi.nlm.nih.gov/31037960

120. Broekaart DWM, Anink JJ, Baayen JC, Idema S, de Vries HE, et al. Activation of the innate immune system is evident throughout epileptogenesis and is associated with blood-brain barrier dysfunction and seizure progression. Epilepsia. 2018; 59: 1931-1944. PubMed: https://pubmed.ncbi.nlm.nih.gov/30194729/

121. Greene C, Kealy J, Humphries MM, et al. Dose-dependent expression of claudin- 5 is a modifying factor in schizophrenia. Mol Psychiatry. 2018; 23: 2156-2166.

PubMed: https://pubmed.ncbi.nlm.nih.gov/28993710/

122. Maes M, Vojdani A, Geffard M, et al. Schizophrenia phenomenology comprises a bifactorial general severity and a single-group factor, which are differently associated with neurotoxic immune and immuneregulatory pathways. Biomol Concepts. 2019; 10: 209-225.

PubMed: https://pubmed.ncbi.nlm.nih.gov/31734647

123. Maes M, Sirivichayakul S, Kanchanatawan B, Vodjani A. Breakdown of the Paracellular Tight and Adherens Junctions in the Gut and Blood Brain Barrier and Damage to the Vascular Barrier in Patients with Deficit Schizophrenia. Neurotox Res. 2019; 36: 306-322. PubMed: https://pubmed.ncbi.nlm.nih.gov/31077000/

124. Raza MW, Shad A, Pedler SJ, Karamat KA. Penetration and activity of antibiotics in brain abscess. J Coll Physicians Surg Pak. 2005; 15: 165-167.

PubMed: https://www.ncbi.nlm.nih.gov/m/pubmed/15808097

125. Yang RC, Qu XY, Xiao SY, Li L, Xu BJ, et al. Meningitic Escherichia coli-induced upregulation of PDGF-B and ICAM-1 aggravates blood-brain barrier disruption and neuroinflammatory response. J Neuroinflammation. 2019; 16: 101.

PubMed: https://pubmed.ncbi.nlm.nih.gov/31092253/

126. Ma Z, Peng J, Yu D, Park JS, Lin H, et al. A streptococcal Fic domaincontaining protein disrupts blood-brain barrier integrity by activating moesin in endothelial cells. PLoS Pathog. 2019; 15: e1007737. PubMed: https://pubmed.ncbi.nlm.nih.gov/31071198/

127. Chen J, Li N, Wang B, Liu X, Liu J, et al. Upregulation of CBP by PLY can cause permeability of blood-brain barrier to increase meningitis. $\mathrm{J}$ Biochem Mol Toxicol. 2019; 33: e22333.

PubMed: https://pubmed.ncbi.nlm.nih.gov/30980515/

128. Thorsdottir S, Henriques-Normark B, lovino F. The Role of Microglia in Bacterial Meningitis: Inflammatory Response, Experimental Models and New Neuroprotective Therapeutic Strategies. Front Microbiol. 2019; 10: 576.

PubMed: https://www.ncbi.nlm.nih.gov/pmc/articles/PMC6442515/

129. Silwedel C, Haarmann A, Fehrholz M, Claus H, Speer CP, et al. More than just inflammation: Ureaplasma species induce apoptosis in human brain microvascular endothelial cells. J Neuroinflammation. 2019; 16: 38.

PubMed: https://pubmed.ncbi.nlm.nih.gov/30764830/ 